\title{
HPV Vaccine Status and BMI Correlation
}

\author{
Ezhilarasi Manickavasagam ${ }^{1 *}$, Hongying Dai ${ }^{2}$ and Joan R Griffith ${ }^{3}$ \\ ${ }^{1}$ General Pediatrics Resident, University of Toledo, Toledo, Ohio, USA \\ ${ }^{2}$ Department of Research Development and Clinical Investigation, Children's Mercy Hospital, Kansas City, Missouri, USA \\ ${ }^{3}$ General Academic Pediatrics, University of Toledo, Toledo, Ohio, USA
}

Received: December 04, 2013; Accepted: February 26, 2014; Published: February 27, 2014

“Corresponding author: Ezhilarasi Manickavasagam, 702 Martin Luther King Road, Mound Bayou, MS-38762, USA, Tel: 662-741-8861; Fax: 662-7418896; E-mail: ezhil.naren@gmail.com

\begin{abstract}
Obese women are at increased risk of death from cervical cancer compared to non-obese women. Yet, research indicates a lower rate of cervical cancer screening among obese women, especially severely obese white women. Cited reasons for delaying screening include embarrassment, perceived weight stigma, lack of appropriately sized examination equipment and poor patient-provider communication. Parental acceptance of the Human Papillomavirus (HPV) vaccine for overweight adolescent females may decrease the incidence of cervical cancer among obese women in later years. This study seeks to determine the Human Papillomavirus (HPV) vaccination status of females, ages 11 to 21 years, in a university-based pediatric practice and to determine the correlation between HPV vaccine status and body mass index (BMI).
\end{abstract}

Methods: We reviewed 800 randomly selected paper medical charts of females, ages 11 to 21 years, enrolled in a university-based pediatric practice as of October 31,2008. Data collected included age, height, weight, BMI, race, insurance carrier, immunizations, sexually transmitted infections (STIs) and documentation of STI prevention counseling. Statistical analysis was performed to determine associations among risk factors and outcome variables.

Results: 800 charts reviewed; 91 were incomplete due to missing documentation of immunizations. The remaining 709 charts included 338 (48.2\%) African Americans; 302 (43.1\%) Whites; 26 (3.7\%) Hispanics; and 35 (5\%) other. Approximately $28.2 \%$ had current HPV vaccine status. There was no correlation between HPV vaccination status and BMI ( $p>0.05)$.

Conclusions: Our study fails to demonstrate higher HPV current vaccination status with increasing BMI. Healthcare providers should continue to encourage HPV vaccination.

Keywords: BMI; Cervical cancer; Health promotion; Human papillomavirus vaccination; Obesity; Risk-taking behavior

\section{HPV Vaccine Status and BMI Correlation}

Age-appropriate vaccinations have proven to be successful public health measures to prevent and control infectious diseases. High school students with extreme obesity participate in risk-taking behavior (such as unprotected sexual activity, substance abuse, and drinking) similar to healthy weight peers [1]. Overweight/ obese adolescents represent an at-risk group for cervical cancer in later years and possible underutilization of HPV vaccination.
Several factors influence parental acceptance of vaccines including, understanding of the health consequences of diseases; knowledge of vaccine benefits and risks; influence of media and celebrities; and other social, behavioral and environmental variables [2]. Although parental concern that HPV vaccine may lead to promiscuity has not been substantiated [3], HPV vaccination rates among adolescents remain low. In 2010, the Centers for Disease Control estimated that $48.7 \%$ of 13 - to 17 year-olds had received one dose the HPV vaccine and $32 \%$ had received all 3 doses versus $44.3 \%$ and $26.7 \%$ respectively in 2009 [4].

Infection with HPV 16 and 18 is the most significant risk/ causative factor in cervical cancer etiology [5]. The incidence of cervical cancer among obese women is higher compared to nonobese women [6]. Since childhood overweight tracks into young adulthood [7], increasing HPV vaccination among adolescents is warranted in view of the finding that disparities in cervical cancer screening by body weight persist for white women with severe obesity [8]. Reduced cervical cancer among obese women could be achieved if we improved compliance rates for HPV vaccination among adolescent females, especially overweight/ obese adolescents.

This study seeks to determine the HPV vaccination status of females, ages 11 to 21 years, in the University of Toledo pediatric practice and to determine the correlation between HPV vaccine status and body mass index (BMI). Since overweight/obese females are at increased risk of cervical cancer, our research question is whether HPV vaccination status increases with increasing BMI.

\section{Methods \\ Participants}

The study took place at the University of Toledo, Toledo, Ohio. The pediatric outpatient practice enrolled patients from birth until age 21 year and the study population is representative of the Lucas County population based on the 2009 census. The University of Toledo outpatient pediatric practice recommends the HPV vaccine at age 11 years. Medicaid covers the HPV vaccine for ages 9 thru 18 years and the commercial insurers cover the 
vaccine for ages 9 thru 26 years or ages 8 thru 27 years. After approval by the University of Toledo Institutional Review Board (IRB) the study was started as the scholarly project by one of the authors who was a third year pediatric resident (EM) and her faculty mentor (JRG). The other author (HD) was contracted as the statistician and has collaborated with JRG on previous research studies.

A representative from the pediatric billing staff generated a computerized list of female patients, ages 11 to 21 years, who were enrolled in the practice as of October 31,2008 . We randomly selected 800 of these patients. Their paper charts were reviewed between January 2009 and October 31, 2009 by one author (EM) using an IRB-approved data collection form. To protect patient confidentiality, all charts were assigned a random number code. The key for the codes was maintained by the PI (EM). The IRB-approved data collection form used to review the charts included age; height; weight and BMI $(\mathrm{kg} / \mathrm{m} 2)$ as recorded on the most recent physical exam; race/ethnicity; documentation of vaccines recorded on the certificate of immunization compliance form; parental refusal of vaccines; documentation of sexual activity status; documentation of positive test(s) for Neisseria gonorrhoeae (GC), Chlamydia trachomatis, Herpes simplex, Rapid Plasma Reagin (RPR), and/or Human immunodeficiency virus (HIV); documentation of sexually transmitted infection prevention counseling; any recommendations for additional sexually transmitted infection screening; and type of medical insurance. Vaccination status was deemed "current" if the ageappropriate number of shots, based on 2009 Centers for Disease Control Advisory Committee on Immunizations Practices (ACIP) recommendations, was documented. The data was analyzed using Fisher's exact test, Pearson Chi-square test, logistic regression, and general linear model.

\section{Statistics}

Statistical analysis was performed using SAS 9.2 and SPSS 18. Pearson chi-square test, Fisher's exact test, logistic regression and general linear model were applied to evaluate the associations among risk factors and outcome variables. Kappa coefficients were determined to evaluate degrees of agreement between vaccines. Cochran-Armitage trend test was applied to relate the number of HPV vaccinations with history of STI. Statistical significance was claimed at $95 \%$ confidence interval $(p<0.05)$.

\section{Results}

Of the 800 charts reviewed, 91 charts did not have documentation of immunization and were removed from further analysis. The remaining 709 charts included 338 (48.2\%) African Americans; 302 (43.1\%) Whites; 26 (3.7\%) Hispanics, and 35 (5\%) others. Subjects were between 11 and 21 years old with mean age $14.9 \pm 2.4$ years old. The study population consisted of $16.1 \%(106 / 658)$ overweight subjects (BMI between $85^{\text {th }}$ and $95^{\text {th }}$ percentile) and $22.1 \%(146 / 658)$ obese subjects (BMI greater than the $95^{\text {th }}$ percentile). BMI percentile was not associated with HPV immunization status or type of insurance. Overweight and obese subjects were more likely to have a history of STI as compared to normal weight subjects. History of STI was reported in $19.4 \%$ of overweight subjects and $19.8 \%$ of obese subjects versus $12.1 \%$ of normal weight subjects $(\mathrm{p}=0.04)$. There was an increasing trend of sexual activity relative to BMI status. Sexual activity was reported in $47.9 \%$ of normal weight subjects, $51.3 \%$ of overweight subjects and $57.0 \%$ of obese subjects ( $p=0.09$ ). There was no association between HPV vaccination status and sexual activity $(>0.05)$.

Whites subjects were more likely to have current HPV status as compared to other subjects (Whites 32\%; African American 29\%; Hispanics 8\%; other $10 \%\{\mathrm{p}=0.0085\}$ ). African American subjects had a higher rate of sexual activity (Whites 40\%; African American 61\%; Hispanic 40\%; other 32\% $\{\mathrm{p}=0.0001\}$ ) and history of STI (Whites 6\%, African American 24\%, Hispanics 8\%, other $4 \%\{\mathrm{p}=0.0001\}$ ). The definition of immunization status along with the distribution of vaccine shots are listed in Table 1. Only $1.5 \%(10 / 687)$ of parents refused vaccination for their child. The reason for refusal was not documented. Approximately $28.2 \%(192 / 680)$ of subjects completed all three doses of HPV vaccines. The highest proportion of current status was noted for MMR (619/654 = 94.7\%). HPV vaccine had slight agreement with Menactra (Kappa $=0.143 ; \mathrm{p}<0.0001)$ and Tdap (Kappa $=0.08 ; \mathrm{p}$ $=0.0299$ ) (Table 2). Subjects with current HPV status were more likely to have current Menactra (96\% vs. $75 \%$ ) and Tdap (52\% versus $43 \%$ ) as compared to subjects without current HPV status. Approximately $45.2 \%$ of subjects had concordant immunization status between HPV and Menactra. Only 55.9\% of subjects had concordant HPV and Tdap current status. The discordance was due to a higher Tdap vaccination rate than HPV rate. HepB had general agreement with DTap, IPV, HIB, and Menactra.

A history of STI was correlated with current status of HPV (Table 3). Subjects with documentation of STI were trended to have more shots of HPV (Cochran-Armitage trend test, $\mathrm{p}$-value $=0.0041$ ) and were more likely to have current status of HPV (43.5\% vs. $26.1 \%, p=0.005)$. Thirty-five percent $(233 / 636)$ of subjects with documentation of insurance had commercial insurance and 65\% (432/636) had Medicaid insurance. There was no correlation between insurance and HPV vaccination status.

\section{Discussion}

In this single center, retrospective chart review, there was no association between HPV vaccination status and BMI. Based on a limited literature review (Pubmed and Pubmed health), we were unable to identify any previous publication studying the relationship between HPV vaccine status and BMI. (Keywords used were 'HPV vaccination and obesity'; 'HPV vaccination and body mass index'; 'Vaccination and obesity'; 'vaccination and body mass index'; 'Human papilloma virus and obesity'). This lack of data suggests that additional research in this area is warranted to determine if our findings are corroborated by future studies.

We also found no association between HPV vaccination status and type of insurance. Zimet et al [9] found that parents with less education, those with Medicaid insurance, and those attending an urban clinic were more likely to be high vaccine acceptors. In contrast, other studies have indicated that these same factors 
Table 1: Status and Distribution of Vaccination.

\begin{tabular}{|c|c|c|c|c|c|c|c|c|c|c|}
\hline Vaccine & $\begin{array}{c}\text { Current } \\
\text { Definition }\end{array}$ & $\begin{array}{l}\text { Current } \\
\text { Status }\end{array}$ & O Shot & 1 Shot & 2 Shots & 3 Shots & 4 Shots & 5 Shots & 6 Shots & 7 Shots \\
\hline Hep B & 3 or more shots & $\begin{array}{c}627 \\
(96.5 \%)\end{array}$ & $9(1.4 \%)$ & $6(0.9 \%)$ & $8(1.2 \%)$ & $\begin{array}{c}625 \\
(96.2 \%)\end{array}$ & $2(0.3 \%)$ & & & \\
\hline MMR & 2 shots & $\begin{array}{c}619 \\
(94.7 \%)\end{array}$ & $10(1.5 \%)$ & $25(3.8 \%)$ & $\begin{array}{c}619 \\
(94.7 \%)\end{array}$ & & & & & \\
\hline IPV & 4 or more shots & $\begin{array}{c}598 \\
(91.9 \%)\end{array}$ & 7 (1.1\%) & 3 (0.5\%) & $2(0.3 \%)$ & $41(6.3 \%)$ & $\begin{array}{c}597 \\
(91.7 \%)\end{array}$ & $1(0.2 \%)$ & & \\
\hline Meningococcal & 1 shot & $\begin{array}{c}543 \\
(80.9 \%)\end{array}$ & $\begin{array}{c}130 \\
(19.3 \%)\end{array}$ & $\begin{array}{c}543 \\
(80.9 \%)\end{array}$ & & & & & & \\
\hline HPV & 3 shots & $\begin{array}{c}192 \\
(28.2 \%)\end{array}$ & $\begin{array}{c}253 \\
(37.2 \%)\end{array}$ & $\begin{array}{c}118 \\
(17.4 \%)\end{array}$ & $\begin{array}{c}117 \\
(17.3 \%)\end{array}$ & $\begin{array}{c}192 \\
(28.2 \%)\end{array}$ & & & & \\
\hline Varicella & $\begin{array}{c}2 \text { shots or had the } \\
\text { disease }\end{array}$ & $\begin{array}{c}201 \\
(31.9 \%)\end{array}$ & $\begin{array}{c}174 \\
(27.6 \%)\end{array}$ & $\begin{array}{c}256 \\
(40.6 \%)\end{array}$ & $72(11.4 \%)$ & & & & & \\
\hline HIB & 4 or more shots & $\begin{array}{c}353 \\
(54.2 \%)\end{array}$ & $54(8.3 \%)$ & $\begin{array}{c}100 \\
(15.4 \%)\end{array}$ & $34(5.2 \%)$ & $\begin{array}{c}110 \\
(16.9 \%)\end{array}$ & $\begin{array}{c}349 \\
(53.6 \%)\end{array}$ & $1(0.2 \%)$ & $3(0.5 \%)$ & \\
\hline DTAP/Tdap & 6 or more shots & $\begin{array}{c}301 \\
(44.1 \%)\end{array}$ & $2(0.3 \%)$ & $33(4.8 \%)$ & $3(0.4 \%)$ & $8(1.2 \%)$ & 47 (6.9\%) & $\begin{array}{c}288 \\
(42.3 \%)\end{array}$ & $\begin{array}{c}300 \\
(44.0 \%)\end{array}$ & $1(0.2 \%)$ \\
\hline
\end{tabular}

Table 2: Kappa Agreement Measures of Current Vaccine Status.

\begin{tabular}{|c|c|c|c|c|c|c|c|}
\hline & HepB & Dtap & IPV & HIB & Meningoccal & MMR & Varicella (31.9\%) \\
\hline HPV (28.2\%) & $0.02^{*}$ & $0.08^{*}$ & 0.02 & -0.01 & $0.143^{* *}$ & 0.01 & $0.13^{* *}$ \\
\hline HepB (96.5\%) & & $0.04^{* *}$ & $0.29^{* *}$ & $0.04^{*}$ & $0.08^{* *}$ & $0.30^{* *}$ & $0.02^{*}$ \\
\hline Dtap (44.1\%) & & & $0.09^{* *}$ & 0.03 & $0.19^{* *}$ & $0.06^{* *}$ & $0.08^{*}$ \\
\hline IPV (91.9\%) & & & & $0.09^{* *}$ & 0.05 & $0.43^{* *}$ & -0.12 \\
\hline HIB (54.2\%) & & & & & -0.07 & 0 & $0.06^{*}$ \\
\hline Meningoccal (80.9\%) & & & & & & & $0.10^{*}$ \\
\hline MMR (94.7\%) & & & & & & & 0.01 \\
\hline
\end{tabular}

\& Kappa coefficient guide: <0 no agreement; 0-0.2 slight agreement; 0.21-0.4 fair agreement;

0.41-0.6 moderate agreement; $0.61-0.8$ substantial agreement; $0.81-1.0$ almost perfect agreement

$*$ p-value $<0.05$

**p-value $<0.01$

Table 3: History of STI Data.

\begin{tabular}{|c|c|c|c|}
\hline \multirow{2}{*}{ Variable } & \multicolumn{2}{|c|}{ History of STI } & \multirow{2}{*}{ p-value } \\
\cline { 2 - 3 } & No $(\mathrm{n}=378)$ & Yes $(\mathrm{n}=69)$ & $<0.0001$ \\
\hline Age & $15.2 \pm 2.2^{*}$ & $17.4 \pm 1.7$ & \multirow{2}{*}{$<0.0001$} \\
\hline White & $163(43.5 \%)$ & $10(14.5 \%)$ & \multirow{2}{*}{. } \\
\hline African American & $178(47.5 \%)$ & $57(82.6 \%)$ & \multirow{2}{*}{0.0052} \\
\hline Hispanic & $11(2.9 \%)$ & $1(1.45 \%)$ & \multirow{2}{*}{$\mathrm{P}=0.04$ (overweight and obese vs. normal weight) } \\
\hline Normal Weight & $232(62.5 \%)$ & $32(48.5 \%)$ & $<0.0001$ \\
\hline Overweight & $54(14.6 \%)$ & $32(48.5 \%)$ & $<0.0001$ \\
\hline Obese & $85(22.9 \%)$ & $21(31.8 \%)$ & \\
\hline HPV Current & $99(26.1 \%)$ & $30(43.5 \%)$ & Not Significant \\
\hline Counseling about safe Sex & $207(54.9 \%)$ & $67(97.1 \%)$ & \\
\hline Offering Additional STI Testing & $108 / 217(49.8 \%)$ & $66 / 68(97.1 \%)$ & Not Significant \\
\hline Medicaid & $231(64.9 \%)$ & $47(71.2 \%)$ & \\
\hline Commercial Insurance & $125(35.1 \%)$ & $19(28.8 \%)$ & \\
\hline
\end{tabular}

*Mean \pm standard deviation (std)

were associated with under immunization of young children [9].

Zimet et al [9] noted that sexually inexperienced individuals were less likely to accept the HPV vaccine. Our findings were similar as we did not demonstrate an increased risk of sexual activity with HPV vaccination. We observed an increased risk of STI among subjects with current HPV vaccination status compared to those who were not current; however, this finding should be interpreted with caution since over a third of the charts lacked STI information.

Our finding of a correlation between BMI and STI disagreed 
with Wee $[10,11]$ who determined 1 ) that after adjustment for age, race/ethnicity, and education, women with higher BMI were less likely to report sexual behavioral risk factors than normalweight women; and 2) that HPV and sexual behavioral risk factors for HPV and cervical cancer were not more prevalent in obese than normal-weight women.

Our study demonstrated a trend of increased sexual activity and increased STI as BMI increased. This finding differs slightly from Kershaw [12] who concluded that overweight women were more likely to have an STI than normal weight women; however, obese women were less likely to have an STI compared to normal weight women. This disagreement could reflect less welldeveloped decision making by our subjects as the average age of our subjects was 14 years as well as the fact that sexual activity among adolescents typically increases with age as does BMI.

There were two major limitations of the study. First, it was a retrospective study and we had to rely on others for accurate record-keeping. Secondly, it was a single center study of a practice consisting of more than $70 \%$ Medicaid patients and our results may not generalize to practices with a significantly different payer mix. The strength of the study was the large sample size and comprehensive look at adolescent immunizations in our practice.

Our findings documented inadequate HPV vaccine status among overweight/obese adolescents. Cervical cancer incidence is higher in overweight/obese individuals. Hence these individuals should be targeted more for HPV vaccination. We recommend health care providers educate their adolescent patients and parents on the increased risk of cervical cancer especially among overweight/obese females as a means to improve HPV vaccination compliance, and subsequently reduce the incidence of cervical cancer in this at-risk population.

\section{Acknowledgment}

We thank the medical staff who helped retrieve the patients charts.

\section{References}

1. Ratcliff MB, Jenkins TM, Peiter-Purtill J, Noll JG, Zeller MH (2011) Risktaking behaviors of adolescents with extreme obesity: normative or not? Pediatrics 127(5): 827-834.

2. Kimmel SR, Burns IT, Wolfe RM, Zimmerman RK (2007) Addressing immunization barriers, benefits, and risks. J Fam Pract 56(2): S61-S69.

3. Bednarczyk RA, Davis R, Ault K, Orenstein W (2012) Sexual activityrelated outcomes after human papillomavirus vaccination of 11 - to 12-year-olds. Pediatrics 30: 798-805.

4. Centers for Disease Control and Prevention (CDC) (2011) National and state vaccination coverage among adolescents aged 13 through 17 years---United States, 2010. MMWR 60(33): 1117-1123.

5. Ghazal-Aswad, S. (2008) Cervical cancer prevention in the human papilloma virus vaccine era. Ann N Y Acad Sci 1138: 253-256.

6. Maruthur NM, Bolen S, Brancati FL, Clark JM (2009) The association of obesity and cervical cancer screening: a systematic review and metaanalysis. Obesity (Silver Spring) 17(2): 375-381.

7. Deshmukh-Taskar P, Nicklas TA, Yang S-J, Zakeri I, et al. (2006) Tracking of overweight status from childhood to young adulthood: the Bogalusa Heart Study. Eur J Clin Nutr 60(1): 48-57.

8. Aldrich T, Hackley B (2010) The impact of obesity on gynecologic cancer screening: an integrative literature review. J Midwifery Womens Health 55(4): 344-356.

9. Zimet GD, Mays RM, Sturm LA, Ravert AA, Perkins SM, et al. (2005) Parental attitudes about sexually transmitted infection vaccination for their adolescent children. Arch Pediatr Adolesc Med 159(2): 132-137.

10. Wee CC, Huang A, Juskey KW, McCarthy EP. (2008) Obesity and the likelihood of sexual behavioral risk factors for HPV and cervical cancer. Obesity 16(11): 2552-2555.

11. Wee CC, Phillips RS \& McCarthy EP (2005) BMI and cervical screening among white, African-American, and Hispanic women in the United States. Obes Res 13(7): 1275-1280.

12. Kershaw TS, Arnold A, Lewis JB, Magriples U (2010) The skinny on sexual risk: the effect of BMI on STI incidence and risk. AIDS Behav 15(7): 1527-1538. 